\title{
Low-Coherence Reflectometry and Speckle Polarimetry in the Monitoring of Human Skin Pathologic Changes
}

\author{
Elena M. Artemina ${ }^{1 *}$, Sergey A. Yuvchenko ${ }^{2}$, Marina V. Alonova ${ }^{2}$, Dmitry A. Zimnyakov², \\ and Sergey R. Utz ${ }^{1}$ \\ ${ }^{1}$ Saratov State Medical University named after V.I. Razumovsky, 112 Bolshaya Kazachya Str., Saratov 410012, Russia \\ ${ }^{2}$ Saratov State Technical University named after Yu.A. Gagarin, 77 Politekhnicheskaya Str., Saratov 410054, Russia \\ * e-mail: farfalla87@mail.ru
}

\begin{abstract}
We present the results of the study of normal and pathologic human skin using low-coherence reflectometry and speckle polarimetry. The statistical characteristics of local polarisation states in the individual coherence areas (speckles) of the forward scattered laser radiation demonstrate high sensitivity to the pathologic changes of the biotissue morphology in vitro. The analysis of the attenuation rate of the low-coherence reflectometer signal depending on the probing depth provides additional information for the identification of skin morphologic changes and the analysis of the effect of immersion agents on the biotissue optical properties. (C) 2018 Journal of Biomedical Photonics \& Engineering.
\end{abstract}

Keywords: surface epidermis strip; polarisation diagnostics of biotissues; optical clearing of biotissues; optical coherence tomography.

Paper \#3267 received 18 Dec 2017; revised manuscript received 18 Feb 2018; accepted for publication 18 Feb 2018; published online 20 Mar 2018. doi: 10.18287/JBPE18.04.010501. [Special Section. Workshop "Biophotonics" of the XV all-Russian Youth Samara conference-contest on optics and laser physics].

\section{References}

1. V. V. Tuchin, Handbook of optical biomedical diagnostics, SPIE Press Bellingham, Washington (2016).

2. V. V. Tuchin, Optics of biological tissues. Methods of light scattering in medical diagnostics, FIZMATLIT, Moscow (2012).

3. D. A. Zimnyakov, M. V. Alonova, E. M. Reshetnikova, E. M. Galkina, S. R. Utz, J. S. Sina, O. V. Angelsky, S. B. Ermolenko, and P. V. Ivashko, "Polarization biopsy in the regime of low forward scattering: a comparative analysis of human skin pathologies," Problems of optical physics and biophotonics SMF-2013: mater. XVII International. youth. sci. shc. on optics, laser physics and biophotonics, 17-25 (2013).

4. A. Fercher, "Optical coherence tomography - development, principles, applications," Zeitschrift für Medizinische Physik 20(4), 251-276 (2010).

5. G. Jarry, E. Steimer, V. Damaschini, M. Epifanie, M. Jurczak, and R. Kaiser, "Coherence and polarization of light propagating through scattering media and biological tissues," Applied Optics 37(31), 7357-7367 (1998).

6. J. Briers, "Laser Doppler, speckle and related techniques for blood perfusion mapping and imaging," Physiological measurement 22(4), R35-R66 (2001).

7. T. K. Lee, L. Tchvialeva, I. Markhvida, H. Zeng, H. Lui, A. Doronin, I. Meglinski, and D. I. McLean, "Polarization Speckles and Skin Applications," Imaging in Dermatology, 77-87 (2016).

8. S. L. Jacques, J. C. Ramella-Roman, and K. Lee, "Imaging skin pathology with polarized light," Journal of Biomedical Optics 7(3), 329-340 (2002).

9. S. L. Jacques, M. R. Ostermeyer, L. V. Wang, and D. V. Stephens, "Polarized light transmission through skin using video reflectometry: toward optical tomography of superficial tissue layers," Proceedings of SPIE 2671, 199-211 (1996).

10. S. R. Utz, S. I. Dovganskiy, and O. D. Odoevskaya, "Use of the method of superficial skin biopsy in dermatological practice," Vestnik dermatologii i venerologii 7, 37-38 (1992) [in Russian].

11. P. V. Novitsky, and I. A. Zograf, Estimation of errors in measurement results, 2th Edition, Energoatomizdat Publishing House, Leningrad (1991) [in Russian]. 
12. D. A. Zimnyakov, Yu. P. Sinichkin, P. V. Zakharov, and D. N. Agafonov, "Residual polarization of noncoherently backscattered linearly polarized light: the influence of the anisotropy parameter of the scattering medium," Waves in Random Media 11(4), 395-412 (2001).

13. E. M Artemina, S. R. Utz, S. A. Yuvchenko, D. A. Zimnyakov, and M. V. Alonova, "Comparative evaluation of clarifying agents to improve the quality of the far long-wave ultraviolet therapy chronic dermatosis," Saratov Journal of Medical Scientific Research 12(3), 453-458 (2016) [in Russian].

\section{Introduction}

Optical probing of biological tissues in vivo and in vitro is presently one of the most intensely developed approaches to the characterisation of morphologic and functional condition of biotissues and its change in the course of therapeutic impact. It is traditional to estimate the local values of optical transport parameters of the tissue (the scattering coefficient, the absorption coefficient, the scattering anisotropy parameter) in the probed region [1, 2]. However, in spite of the euphoria related to the possible application of optical radiation to the morphologic and functional diagnostics of biotissues, it is somewhat restricted by the complexity of interpretation of the resulting empirical data. In the case of probing with coherent or partially coherent radiation (e.g., laser polarimetry or optical coherence tomography), the difficulties are caused by the stochastic interference of the probing radiation in the biotissue, which is a multiply scattering randomly inhomogeneous medium [3-5]). The interference gives rise to speckle modulation of the probe radiation that decreases the signal-to-noise ratio in the process of probing and reduces the biotissue probing depth. Note, that the effect of speckle modulation of the probing laser radiation can play not only a negative role, but also a positive one in the optical biomedical diagnostics. A typical example are speckle correlation methods applied to monitor the microhaemodynamics in biotissues $[1,2$, 6, 7-9]. Thus, additional information on the morphologic and functional condition of the biotissue under diagnostics can be extracted not only by analysing the attenuated non-scattered ("coherent") component of the probing radiation and the components caused by low-multiplicity scattering, but also by estimating the statistical and correlation properties of multiply scattered speckle modulated "noise" component.

The aim of the present paper is to develop such approach in application to the monitoring of structural changes of human skin caused by different pathologies using the methods of scanning speckle polarimetry (for skin samples in vitro) and low-coherence reflectometry (for skin in vivo).

\section{Experimental technique}

We carried out the experiments in vitro and in vivo with human skin samples in the normal condition and with different kinds of pathologies. The in vitro samples (surface strips of epidermis (SSE)) were taken in the outpatient and inpatient setting of the Clinic of Skin Diseases, Saratov State Medical University named after
V.I. Razumovsky from 20 patients with different dermatoses (lichen ruber planus, alopecia, discoid lupus erythematosus, psoriasis, demodicosis, scabies) aged 18-60 years, and from 10 healthy persons aged 20-40 years.

To get the SSE we used the technique described in Ref. [10] that comprises several steps. A drop of medical glue was applied to a microscope slide preliminarily washed in the $(1: 1)$ mixture of spirit and ether. The slide was applied to the skin and tightly pressed towards it. In 30 seconds, the slide was carefully removed. The medical autosterile nontoxic Sulphacrylate glue (Institute of Catalysis, Siberian Branch of the Russian Academy of Sciences, Novosibirsk) provided firm adhesion between the slide and the epidermis surface that prevented the skin traumatisation. After stripping, the epidermis fragment appeared to be toughly fixed on the glass forming a firm thin film. The material was multiply taken from the same skin area until the appearance of point bleeding. In the process of the study and after it, no side reactions caused by the use of the glue were revealed.

From 9 to 15 skin strip samples with each type of pathology, 3-5 samples 20-80 $\mu \mathrm{m}$ thick from each person, were prepared. The thickness and the surface area of the samples depended on the specific changes of skin structure caused by the particular pathology, as well as on the localisation of the lesion site and the individual characteristics of the patient. Thus, for the samples of skin affected by psoriasis and discoid lupus erythematosus the thickness amounted to $60-80 \mu \mathrm{m}$, and for those with alopecia it was $20-35 \mu \mathrm{m}$. The rest samples, including healthy skin, possessed the thickness $40-60 \mu \mathrm{m}$. Samples of healthy skin were taken from one zone of the body (the inner side of forearm). The surface area of the samples varied from 0.7 to $2 \mathrm{~cm}^{2}$.

The samples of pathologically changed skin were taken from the patients in the stage of clinical presentations (scabies and demodicosis) or exacerbation of chronical diseases.

A schematic diagram of the laboratory scanning speckle polarimeter is presented in Fig. 1 .

As a source of radiation we used the He-Ne laser 1 GN-2P ( $\lambda=633 \mathrm{~nm}, P=2 \mathrm{~mW}$, linear polarisation). The focusing of laser radiation on the sample and the subsequent collimation of non-scattered component of the probing radiation was implemented using the telescopic system of two microscope objectives $\mathrm{O}_{1}$ and $\mathrm{O}_{2}\left(8^{\times}\right.$and $\left.10^{\times}\right)$with conjugate focal planes, in which the studied sample 4 was placed. The polarisation state of the forward-scattered probing radiation output from 


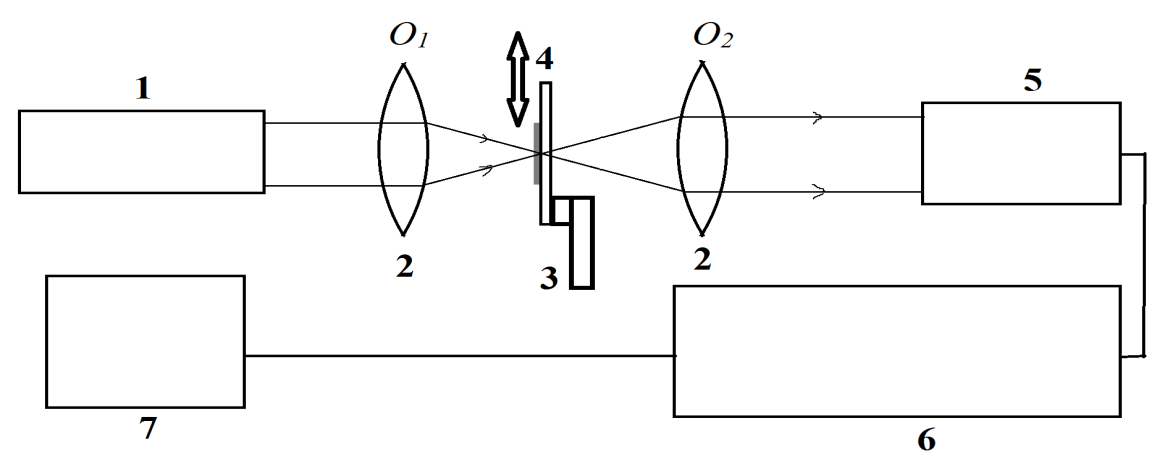

Fig. 1 Schematic diagram of the experimental setup. 1 - He-Ne laser; 2 - telescopic system of two microscope objectives $\mathrm{O}_{1}$ and $\mathrm{O}_{2} ; 3$ - single-coordinate micropositioner; 4 - sample under study; 5 - measuring head of the polarimeter PAN5710VIS; 6 - polarimeter on the platform TXP 5004; 7 - PC.

the microscope objective $\mathrm{O}_{2}$ was analysed using the polarimeter TXP5004 - PAN5710VIS (Thorlabs, USA). The optical field in the receiving aperture of the polarimeter head 5 was a superposition of the attenuated non-scattered component and forward-scattered components with different multiplicity of scattering. The used optical scheme allowed the registration of the probing radiation components scattered forward at small angles to the axis of the laser beam. As a result, in the detection zone large-scale speckles were produced, their mean size being comparable with that of the polarimeter photodetector aperture. The scanning was performed with the step $5 \mu \mathrm{m}$, which in our case provided the statistical independence of local realisations of the scattered field in the detection zone at each step.

In the scanning speckle polarimetry, the values of excentricity $\varepsilon_{i}$ and azimuthal angle $\varphi_{i}$ of the local polarisation ellipses in the analysed region of the scattered light field at each scanning step were used as recorded parameters. The azimuthal angle of the local polarisation ellipse is defined as the angle between the major axis of the ellipse and the polarisation vector in the probing beam. In the used speckle polarimeter the values of the polarisation parameters were determined with the error $\pm 0.01^{\circ}$ for the azimuthal angle and \pm 0.001 for the excentricity. The sets of $\varepsilon_{i}$ and $\varphi_{i}$ values obtained as a result of scanning were subjected to the frequency analysis; besides that using the obtained values the first- and second-order statistical moments were calculated for the excentricity and the azimuthal angle. These parameters were chosen because the obtained empirical distributions of $\varepsilon_{i}$ and $\varphi_{i}$ allow clear identification of the contribution of different processes into the formation of local polarisation states of the detected scattered field. In particular, multiple scattering leads to the homogeneous broadening of the azimuthal angle distribution, if the initial non-scattered probing beam is described by delta-function $\rho(\varphi)=\delta(\varphi)$. At the same time, the presence of structural anisotropy or optically active components in the tissue layer will lead to a shift of the distribution relative to the point $\varphi=0$. Since all obtained results were processed, the possible individual differences were taken into account.

Analogously, the distribution shape for the excentricity of local polarisation ellipses, characterised by negative asymmetry coefficient is determined by the mean multiplicity of scattering of the probing radiation in the tissue layer. The variance of the local excentricity values monotonically grows with the increase of the scattering multiplicity. It is also worth noting that the values of the normalised components of the Stokes vector $s_{1}, s_{2}, s_{3}$, commonly used as detected parameters in the traditional polarimetry of biotissues can be calculated from the sets of $\varepsilon_{i}$ and $\varphi_{i}$ values, obtained in the course of our experiment. On the other hand, the traditional polarimetry based on the characterisation of the probed biotissue using all elements of the Mueller matrix or some part of them, restored from different input and output values of $s_{1}, s_{2}, s_{3}$, is rather labourconsuming and often makes the resulting data interpretation problematic.

In the clinical studies in vivo, the method of lowcoherence reflectometry (LCR) was used. The method is based on the quantitative analysis of the attenuation of the mean signal output from a low-coherence interferometer, used to probe the biotissue, depending on the probing depth. As tools for LCR diagnostics one can use various modifications of optical coherence tomographs. In this case, the analysed signal is formed as a result of averaging over a sample of adjacent Ascans in the zone of probing. In contrast to the traditional OCT diagnostics, mainly based on the qualitative comparison of the OCT images with histologic data, the LCR method, as shown below, implies the comparison of the obtained quantitative characteristics of the damped signal with the data on the structure and optical properties of the tissues.

The LCR probing was conducted both with the diagnostic purpose and with the purpose of choosing an optimal immersion agent for optical clearing of superior skin layers in the phototherapy of lichen ruber planus 
(LRP). 30 patients with manifestations of LRP aged from 18 to 65 years took part in the study. Different clearing agents (glucose, 1,2-propylene glycol, glycerol, oleic acid) were applied to their skin. We have chosen 4 elements in each individual patient, similar in magnitude and size and located in the same anatomic region (the most frequent localisation of skin rash was the flexion surface of forearm). Then, to enhance the penetration of the clearing agent through the stratum corneum, the affected areas were treated with the solution of ethanol. To enhance the transcutaneous penetration and to prevent the draining, the immersion agent was applied to the elements under occlusion (polyethylene film). The LCR probing was carried out in each element in the process of clearing (in 5-15-3060 minutes).

As a low-coherence reflectometer, we used the optical coherence tomograph OSC1300SS with frequency sweeping (Thorlabs, USA) having the central wavelength $1325 \mathrm{~nm}$.

\section{Experimental results and discussion}

In the process of using the scanning speckle polarimetry the sample volumes for the values of excentricity and azimuthal angle of local polarisation ellipses recorded by scanning the skin strips amounted to 1000-2000 values for each skin sample. In the process of the data statistical processing, the frequency analysis was carried out for each scan, after which the obtained distributions of relative frequencies for the values of excentricity and azimuthal angle were averaged over different data sets obtained for one person (see the section "Experimental technique"). In the process of frequency analysis, the number of intervals for grouping the obtained data in the region from the minimal value to the maximal one was chosen to be 18 . This value is somewhat smaller than $\sqrt{N}$ (where $N$ is the sample volume), proposed in Ref. [11] as a rational choice of the optimal number of intervals for the frequency analysis. The number of intervals smaller than $\sqrt{N}$ leads to the coarsening of the obtained histograms, and the greater numbers increase the uncertainty related to the possibility of "empty" intervals. Thus, in our analysis we have made a choice in favour of coarser estimates of the distribution of relative frequencies over the intervals. At the next stage of the analysis, the averaged frequency distributions obtained for each patient with the given pathology were averaged over the group of patients possessing this pathology. Thus, in the course of processing the results of speckle polarimetry, the obtained data were averaged twice, first, for each individual and, second, for the group of individuals. To our opinion, such approach essentially enhances the robustness of the obtained empirical data. Note also that the root-mean-square deviations of the minimal and maximal values of the excentricity and the azimuthal angle from the appropriate mean values for one patient did not exceed $20 \%$. For a group of patients the similar quantity amounted to $32 \%$, which is caused by the spread of individual physiologic features of the patients in the group.

For example, Figs. 2 and 3 present the histograms of the local values of excentricity and azimuthal angles of the polarisation ellipses obtained as described above in the samples of normal skin (a) and pathologic (psoriasis) skin in vitro (b).

In the samples of skin affected by psoriasis (Figs. $2 \mathrm{~b}$ and $3 b$ ) we can see homogeneous broadening of the azimuthal angle distribution with minor shift towards the region of positive values and the monotonic decrease of the relative frequency of excentricity values, the modal value of the relative frequency being equal to 1. These results are in qualitative agreement with the experimental and theoretical data on the transformation of the polarisation state of light, propagating through isotropic multiply scattering media (see, e.g., [12]).

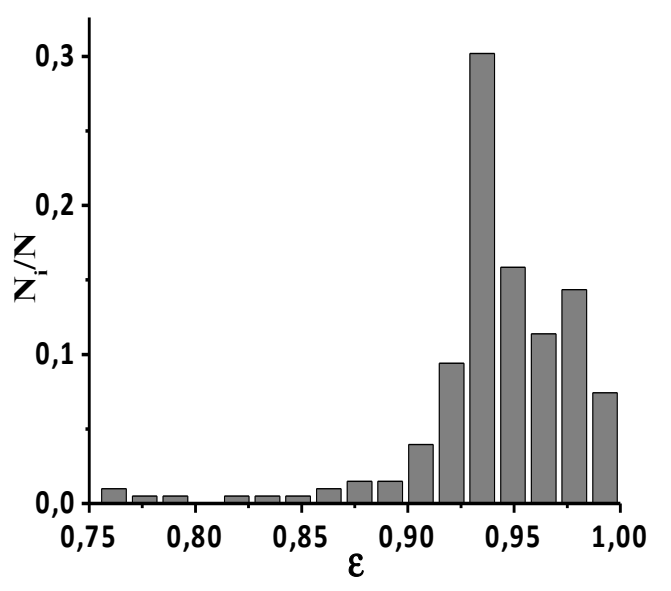

(a)

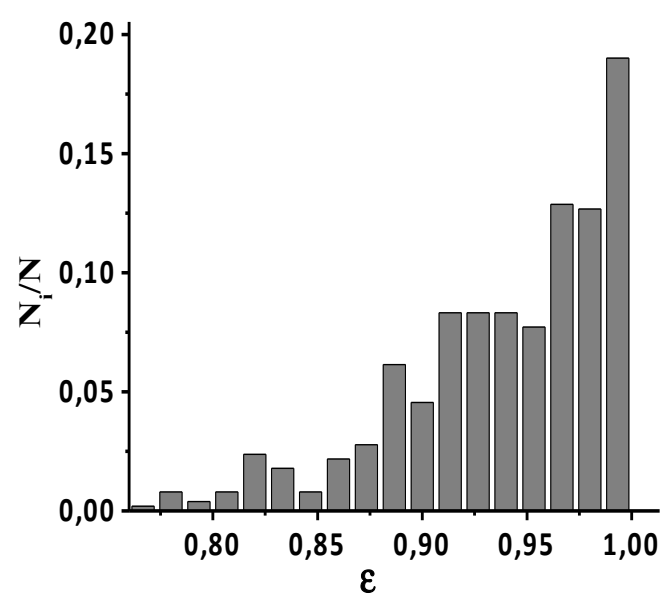

(b)

Fig. 2 Histograms of the local values of the polarisation ellipse excentricity for the epidermis of the in-vitro samples of normal skin (a) and the skin affected by psoriasis (b). 


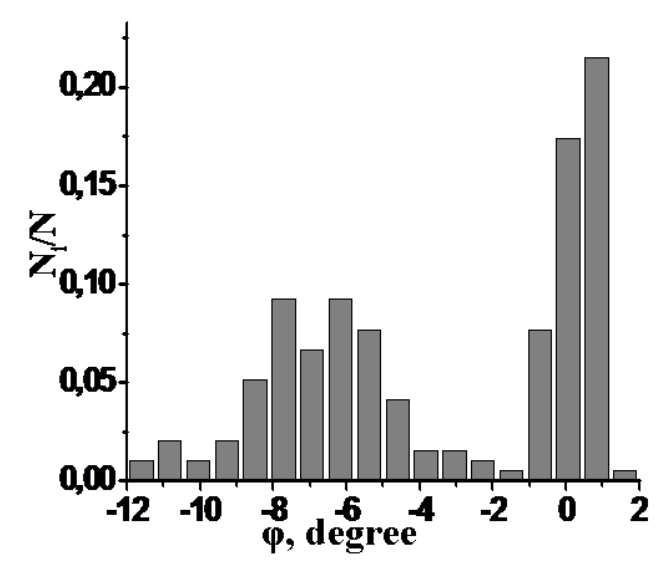

(a)

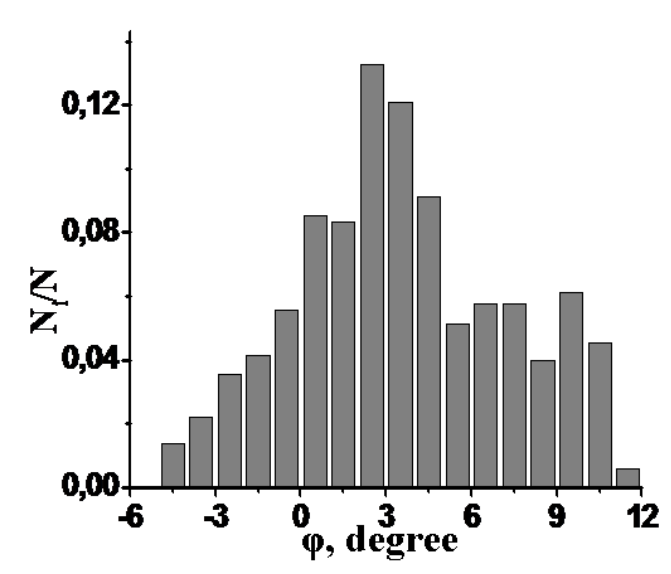

(b)

Fig. 3 Histograms of the local values of the polarisation ellipse azimuthal angle in the epidermis samples of the normal skin in vitro (a) and in the case of psoriasis (b).

On the other hand, one can see qualitative differences of the obtained results for the normal skin, namely, the bimodal character of the azimuthal angle histogram with essential shift of the minor mode towards the region of negative values, as well as the shift of the modal value of the excentricity to the region of small values. These features allow us to suppose that such samples possess a certain type of structural anisotropy. This hypothesis requires additional detailed studies.

As quantitative characteristics of the above peculiarities of the frequency distributions of the local values of the excentricity and the azimuthal angle one can consider the mean values $\langle\varepsilon\rangle$ and $\langle\varphi\rangle$. Using this approach the studied samples of skin strips can be divided into two groups. The first group is characterised by symmetric and asymmetric distributions of the azimuthal angle at small $\langle\varphi\rangle$, and for the second group the negative values of $\langle\varphi\rangle$ with sufficiently large absolute value are typical. The samples of skin affected by alopecia are characterised by considerably expressed bimodality of the azimuthal angle frequency distribution at the maximal absolute value of $\langle\varphi\rangle$. The mean excentricity values for the studied samples lie in the interval from 0.92 to 1 , and the samples can be confidently divided into two groups, with $\langle\varepsilon\rangle>0.96$ and with $\langle\varepsilon\rangle<0.94$.

Such approach to the identification of pathologically affected skin samples in vitro using the scanning speckle polarimetry is illustrated by Fig. 4, where the mapping points corresponding to the normal and pathologic skin samples are presented in the coordinate system "mean azimuthal angle - mean excentricity of polarisation ellipse".

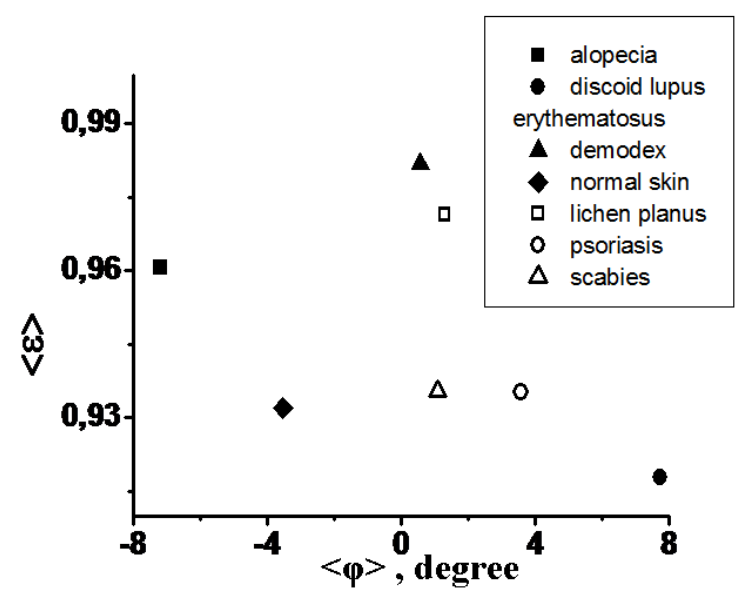

Fig. 4 Mapping of pathologic conditions of human skin epidermis for different diseases in the system of coordinates "mean value of the azimuthal angle - mean value of the polarisation ellipse excentricity" (obtained from the data of speckle polarimetry).

In the case of low-coherence reflectometry of human skin in vivo using the optical coherence tomograph as an instrumental base, the analysis of the recorded OCT signals was performed as follows.

At the first stage for the region chosen for probing we determined the damping LCR signal $I_{r}(z)$ (where $z$ is the probing depth) by averaging over a group of 30 adjacent A-scans recorded by the optical coherence tomograph. As a result of the averaging, the noise speckle modulation of A-scans was reduced to the level acceptable for further analysis. The reconstructed LCR signal is a superposition of diffuse and low-multiplicity scattered components of the probing radiation backscattered by the layer of tissue that can be presented as

$$
I_{r}(z) \approx I_{d r}(z)+I_{s r}(z)
$$

where $I_{d r}(z)$ is the diffuse signal component, $I_{s r}(z)$ is the low-multiplicity scattered component. The 
theoretical analysis of the dependence of the diffuse component decrease rate on the probing depth, performed within the frameworks of the diffusion approximation of the radiation transport theory, and the comparison of the obtained theoretical data with the dependence of LCR signals on $z$ observed experimentally have led us to the conclusion that under the used conditions of probing $I_{d r}(z)<<I_{s r}(z)$. In turn, the component $I_{s r}(z)$ can be presented in the form

$$
I_{s r}(z) \propto Q_{b s}(z) \exp \left(-2 \bar{\mu}_{t} z\right)
$$

where $Q_{b s}(z)$ is the backscattering efficiency factor of the studied tissue at the depth $z, \overline{\mu_{t}}$ is the biotissue extinction coefficient averaged over the probed volume.

At the next stage of the processing, the exponential trend $\exp \left(-2 \overline{\mu_{t}} z\right)$ was extracted from the LCR signal using the least square method. The value of $\overline{\mu_{t}}$ was determined for the probed region and the trend was removed according to the procedure $I_{r}(z) \exp \left(2 \bar{\mu}_{t} z\right) \propto Q_{b s}(z)$. The obtained function is proportional to the depth distribution of the backscattering efficiency factor and can be used both for the quantitative analysis of morphologic features of the biological tissue and for its visualisation. Note that the value of $\overline{\mu_{t}}$ obtained as a result of trend determination can be also used for the quantitative characterisation of the biotissue in the region of probing.

The application of this approach using the LCR probing of skin affected by lichen ruber planus with tissue clearing by means of 1,2-propylene glycol and without it is illustrated in Fig. 5. Figure 6 presents the depth distributions of LCR signals for the affected skin region before the beginning of optical immersion and in 30 minutes after the application of the immersion agent with the corresponding exponential trends. The immersion was implemented by applying the agent to the surface of the affected region. Figure 6,b shows the reconstructed distributions of the normalised values of the backscattering efficiency factor $Q_{b s}(z)$ (the values were normalised to the maximal value of $Q_{b s}(z)$ in the probed region before the application of the immersion agent). In the reconstructed distributions $Q_{b s}(z)$ one can clearly identify two zones associated with the epidermis and the papillary layer of dermis, characterised by more intense backscattering (at the depths from $\approx 100 \mu \mathrm{m}$ to $\approx 350 \mu \mathrm{m}$ and from $\approx 400 \mu \mathrm{m}$ to $\approx 650 \mu \mathrm{m}$ ).

One more example of the application of the developed approach is the estimation of the efficiency of the skin optical clearing in vivo for different biologically harmless immersion agents. In the present case, we estimated the values of $\bar{\mu}$ that determine the exponential trend of the recorded LCR signals at different moments of the immersion agent impact. 30 patients with lichen ruber planus took part in the experiment with each immersion agent. Since the values of $\bar{\mu}_{t}$ obtained from the LCR probing at the initial moment of the optical immersion in different patients lied in the interval from $80 \mathrm{~cm}^{-1}$ to $120 \mathrm{~cm}^{-1}$ (depending on the age, sex and other factors), for the characterisation of the immersion efficiency we used the normalised values $\bar{\mu}_{t}(t) / \bar{\mu}_{t}(0)$, averaged over a group of patients. Figure 6 presents the results of the study of the efficiency of immersion agents that allowed the choice of 1,2-propylene glycol as the most efficient clearing agent for the phototherapeutic applications in dermatology.

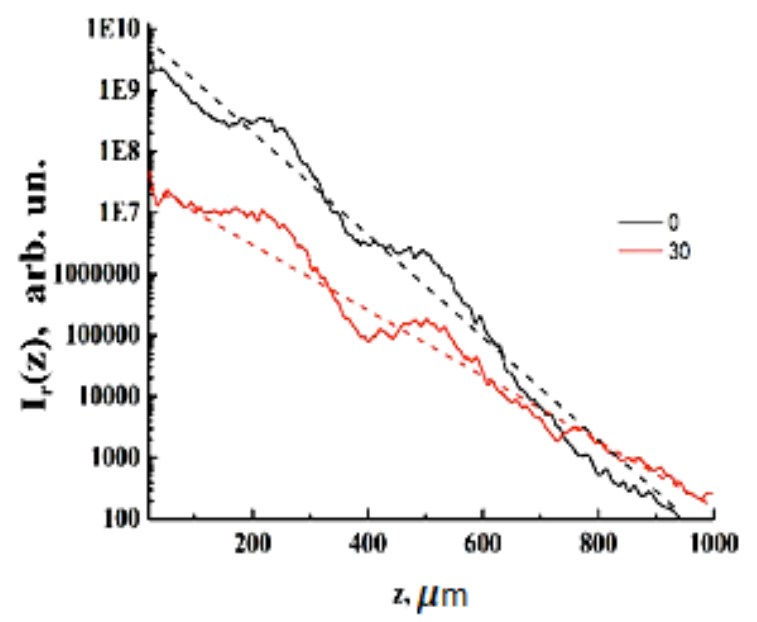

(a)

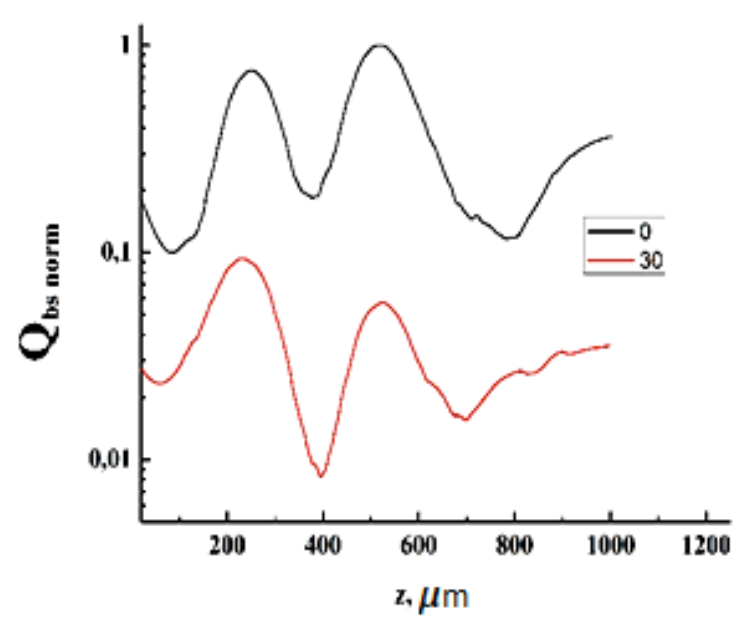

(b)

Fig. 5 The recorded LCR signal (a) and the normalised backscattering efficiency factor (b) versus the probing depth for the skin in vivo affected by the lichen ruber planus. The measurements were executed before the application of 1,2-propylene glycol as immersion agent and in 30 minutes after it. 


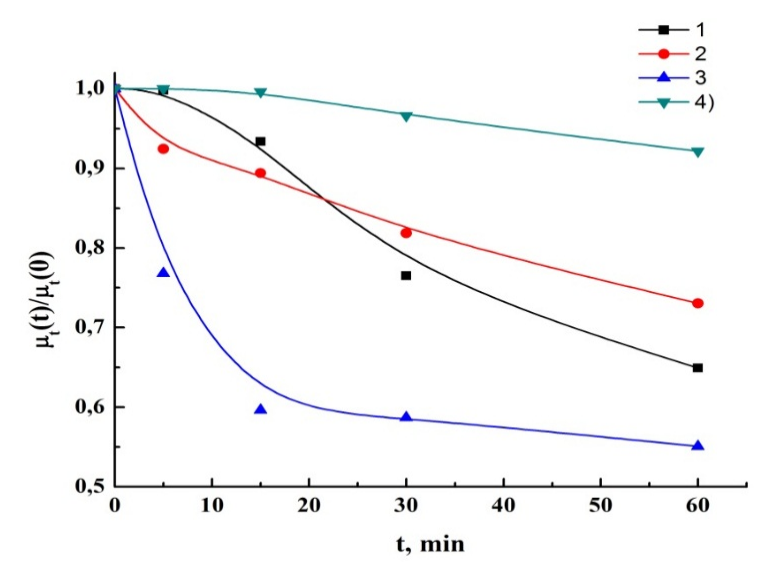

Fig. 6 Dependences of the mean normalised extinction coefficient of human skin in vivo (LRP) on the time of exposure to different immersion agents: 1 - glycerol, 2 - glucose, 3 - 1,2-propylene glycol, 4 - oleic acid. To plot the dependences the obtained data were interpolated by B-splines.

\section{Conclusion}

It should be emphasised that the performed in vitro scanning speckle polarimetry was a pilot study aimed at evaluating the applicability of the method to the identification and characterisation of skin pathologies. Other researchers have also discussed the idea of analysing the polarisation characteristics of multiply scattered speckle-modulated laser radiation for the diagnostics of skin pathologies (in particular, oncologic diseases). In this connection, we should mention the studies by T. Lee et al. from the University of British Columbia in Vancouver (see, e.g., [7]). However, the approaches to the interpretation of the obtained data proposed by this group are ambiguous and do not allow for all specific features of the polarisation state evolution in the process of laser radiation propagation through randomly inhomogeneous multiply scattering media.

As to our results obtained using the discussed method of scanning speckle polarimetry of skin samples in vitro in the transmitted light, we should note that the comparison of the mean values of excentricity and azimuthal angle of the local polarisation ellipse in the forward-scattered light allows the division of samples into a few groups, presumably related to their morphology. We present the preliminary results of mapping different pathologies on the 2D space of diagnostic parameters, chosen as the first-order statistical moments (mean values) of the excentricity and azimuthal angle of the local polarisation ellipses. These results have demonstrated acceptable selectivity of the approach in the identification of different skin pathologies. Additional facilities can be offered by multidimensional mapping of pathologies in the space of diagnostic parameters, which can be, e.g., the higherorder statistical moments of the local values of excentricity and azimuthal angle, determined in the speckle polarisation experiment.

The method of low-coherence reflectometry used in the present work implies the use of the primary signals (A-scans) of the optical coherence tomograph in order to obtain the information on the biotissue extinction coefficient averaged over the probed volume and the depth distribution of the backscattering efficiency factor. With this aim the recorded set of A-scans were additionally processed in order to suppress the noise components, to determine and eliminate the exponential trend. Note that the approach is interesting not only from the point of view of identifying human skin pathologic changes, but also from the point of view of applying therapeutic procedures based on near-UV irradiation of pathologic skin areas with preliminary optical clearing by means of biocompatible immersion agents (e.g., in therapy of lichen ruber planus [13]). In particular, the application of the approach to the determination of the most efficient clearing agent for the phototherapy of skin pathologies has shown its high efficiency.

\section{Disclosures}

All authors declare that there is no conflict of interests in this paper. 